\title{
Excited-state spectra at the superfluid-insulator transition out of paired condensates
}

\section{Citation}

Powell, Stephen, and Subir Sachdev. 2007. "Excited-State Spectra at the Superfluid-Insulator Transition out of Paired Condensates." Physical Review A 75 (3). https://doi.org/10.1103/ physreva.75.031601.

\section{Permanent link}

http://nrs.harvard.edu/urn-3:HUL.InstRepos:41417417

\section{Terms of Use}

This article was downloaded from Harvard University's DASH repository, and is made available under the terms and conditions applicable to Other Posted Material, as set forth at http:// nrs.harvard.edu/urn-3:HUL.InstRepos:dash.current.terms-of-use\#LAA

\section{Share Your Story}

The Harvard community has made this article openly available.

Please share how this access benefits you. Submit a story.

Accessibility 


\title{
Excited state spectra at the superfluid-insulator transition out of paired condensates
}

\author{
Stephen Powell \\ Department of Physics, Yale University, New Haven, CT 06520-8120 \\ Subir Sachdev \\ Department of Physics, Harvard University, Cambridge MA 02138
}

(Dated: September 11, 2018)

\begin{abstract}
We describe gapped single-particle and collective excitations across a superfluid to insulator quantum phase transition of particles (bosons or fermions) in a periodic potential, with an even number of particles per unit cell. We demonstrate that the dynamics is controlled by a quantum impurity problem of a localized particle interacting with the bulk critical modes. Critical exponents are determined by a renormalization group analysis. We discuss applications to spin oscillations of ultracold atoms in optical lattices, and to the electronic phases in the cuprate and related compounds.

PACS numbers: 05.30.-d, 03.75.Kk, 71.10.-w
\end{abstract}

A number of experiments have observed coherent spin oscillations of trapped, ultracold spinor bosonic atoms. With a superfluid ground state [1, 2, 3, 4, 5, 6], these oscillations are well described by the classical (GrossPitaevski) equations which control the time evolution of the multi-component atomic condensate. Recent experiments [7, 8] have also observed oscillations in a Mott insulating state obtained by placing the atoms in an optical lattice; in this situation, the spin oscillations can be viewed as the Rabi precession [7, 9] between sharp quantum states localized within each minimum of the optical lattice.

In this paper, we investigate the connection between these two disparate pictures of the spin oscillations, by describing ground state spin correlations at the quantum critical point separating the superfluid and insulating phases. Bosonic atoms with total spin $F=1$ and $F=2$ display a remarkably rich variety of superfluid and insulating phases 10, 11, 12, 13, 14], and have a corresponding plethora of universality classes of quantum phase transitions separating such phases. Superfluid-insulator transitions are also possible for paired fermionic atoms in an optical lattice. We defer a more complete classification of the spin dynamics at such transitions to a forthcoming paper. Here we focus on a class, with an even integer number of particles per unit cell, which displays nontrivial collective behavior induced by a strong coupling between the spin excitations and the critical number and phase fluctuations of the superfluid-insulator transition; the class includes both the fermionic and bosonic cases. The problem is mapped exactly onto a quantum 'impurity' problem, which couples a single localized spin excitation (the 'impurity') to the bulk critical modes; a solution using the renormalization group yields new critical exponents and scaling functions. Our theory has some analogies to simpler models of the Kondo and Xray edge effect in metals, and opens the way towards observing strongly-coupled quantum impurity physics in ultracold atom systems.

Our results also apply to the superfluid-insulator transition in electronic systems, with an even integer number of electrons per unit cell. Such a situation can arise in the cuprate or related compounds, with a periodic potential generated spontaneously by 'stripe/checkerboard' or charge density wave order: recent experiments in insulating spin ladder compounds 15] have shown that each unit cell contains a pair of holes. Our results predict the frequency dependence of the electron photoemission spectrum across a superfluid-insulator transition in which the 'stripe' order is present on both sides of the transition; in other words, for a transition between a modulated insulator and a supersolid. The predictions of the spectrum are for gapped single particle excitations at positions of the gap maxima or minima in the Brillouin zone e.g. at the analog of the 'antinodal' points.

We will study superfluid-insulator transitions at which the energy gap to both single-particle and spin excitations remains nonzero at the transition. The superfluid order parameter for the transition is then necessarily a spin singlet. The order parameter also carries a nonzero particle number, or 'charge' $Q$. Here, and henceforth, 'particle' refers to either a single ultracold bosonic or fermionic atom, or an electron (but not a Cooper pair). For $F \neq 0$ particles, this means that the simplest case has an order parameter, $\Psi$, with $Q=2$ and $F=0$, corresponding to the annihilation operator for a spin-singlet pair of particles, e.g. a Cooper pair.

For definiteness, we will develop our results in the context of a Bose-Hubbard model for $F \neq 0$ bosons in an optical lattice, and indicate the generalization to the fermionic case later. For this model, we consider the transition from a 'spin-singlet insulator' (SSI), a Mott insulator with an even number of atoms per lattice site and no spin order, to a 'spin-singlet condensate' (SSC), in which singlet pairs of bosons have condensed, but there is no single boson condensate. We will begin with a simple 
mean-field theory of the Bose-Hubbard model, and then turn to a field theory of the critical properties of this and the corresponding fermionic model.

The lattice bosons are annihilated with operators $a_{i, m}$ on lattice site $i$ and spin projection $m=-F \ldots F$. The Bose-Hubbard Hamiltonian can then be written as $\mathcal{H}=$ $-t T+V$, where $T$ is the kinetic energy term,

$$
T=\sum_{\langle i, j\rangle, m}\left(a_{i, m}^{\dagger} a_{j, m}+\text { h.c. }\right),
$$

and $V$ is the on-site interaction:

$$
V=\sum_{i}\left[U\left(\hat{n}_{i}-N\right)^{2}+J\left|\mathbf{L}_{i}\right|^{2}\right]
$$

where $\hat{n}_{i}=\sum_{m} a_{m}^{\dagger} a_{m}$ is the boson number operator on site $i$, and $\mathbf{L}_{i}$ is the total spin operator on site $i$. We have made the spin-independent part of the interaction explicitly symmetric around $N$ particles per site. For $F=1$, the final term is the most general spin-dependent interaction, but further terms are necessary for higher spin. To favor spin-singlet pairing in the ground state, we require $N$ to be even, and $J>0$.

In the case when $t=0$, the Hamiltonian is simply a sum of terms acting on a single site, containing only the commuting operators $\hat{n}$ and $|\mathbf{L}|^{2}$. The ground state on each site is therefore a spin singlet of $N$ bosons.

An appropriate mean-field Hamiltonian is $\mathcal{H}_{\mathrm{mf}}=V-$ $T_{\psi}-T_{\Psi}-T_{\Phi}$, where $V$ is the same on-site interaction as in Eq. (2). $T_{\psi}$ is the standard mean-field decoupling of the hopping term, generalized to the case with spin,

$$
T_{\psi}=\sum_{i}\left[\psi_{m} a_{i, m}^{\dagger}+\psi_{m}^{*} a_{i, m}\right]
$$

where $\psi_{m}$ is a (c-number) constant vector, which will be used as a variational parameter. The remaining terms allow for the possibility of a spin-singlet condensate through the parameters $\Psi$ and $\Phi$ :

$$
T_{\Psi}=\sum_{i, m}(-1)^{F+m}\left[\Psi a_{i, m}^{\dagger} a_{i,-m}^{\dagger}+\Psi^{*} a_{i, m} a_{i,-m}\right]
$$

and

$$
T_{\Phi}=\sum_{\langle i, j\rangle, m}(-1)^{F+m}\left[\Phi a_{i, m}^{\dagger} a_{j,-m}^{\dagger}+\Phi^{*} a_{i, m} a_{j,-m}\right],
$$

where the factors of $(-1)^{F+m}$ are Clebsch-Gordan coefficients that cause the boson operators to form spinsinglet pairs.

We now use the ground state of $\mathcal{H}_{\mathrm{mf}}$, which we denote $|\mathrm{mf}\rangle$, as a variational ansatz and define

$$
E_{\mathrm{mf}}\left(\psi_{m}, \Psi, \Phi\right)=\langle\mathrm{mf}|\mathcal{H}| \mathrm{mf}\rangle
$$

which should be minimized by varying the three parameters. If this minimum occurs for vanishing values of all

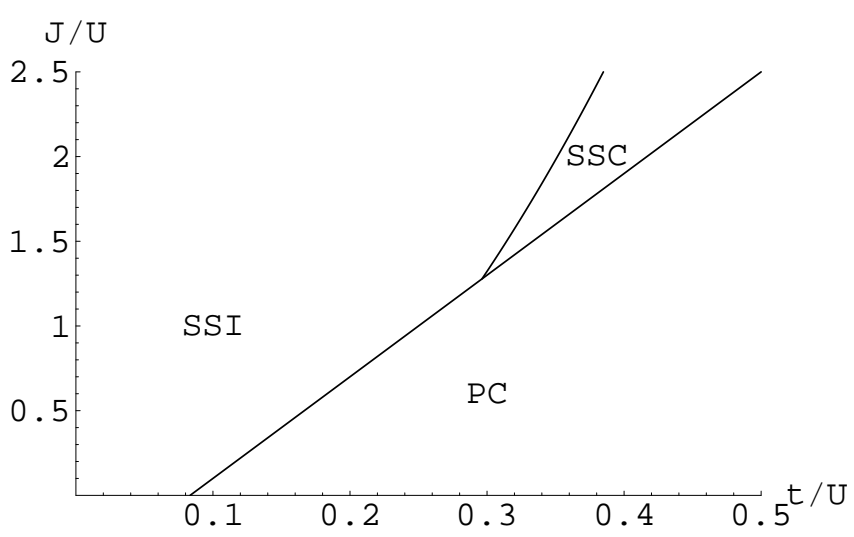

FIG. 1: Phase diagram calculated using mean-field theory. The calculation has been performed for spin $F=1$ and filling factor $N=2$. The horizontal and vertical axes give the tunneling strength $t$ and the spin-dependent interaction $J$, both in units of the spin-independent part of the interaction, $U$.

three parameters, then $|\mathrm{mf}\rangle$ breaks no symmetries and the Mott insulator is favored. A nonzero value for $\psi_{m}$ at the minimum corresponds to a simple 'polar condensate' (PC), breaking spin-rotation symmetry; vanishing $\psi_{m}$ but nonzero values of $\Psi$ and/or $\Phi$ corresponds to a paired spin-singlet condensate.

Since $\mathcal{H}_{\mathrm{mf}}$ contains terms (within $T_{\Phi}$ ) that link adjacent sites, it cannot be straightforwardly diagonalized, as in the standard mean-field theory for the spinless BoseHubbard model. To find the phase boundaries, however, we need only terms up to quadratic order in the variational parameters, which can be found using perturbation theory. Figure 1shows the phase boundaries so obtained.

We now turn to an analysis of the correlation functions of gapped spin-carrying modes across the SSI/SSC transition. We will assume we are in a regime in which the lowest excitation with a nonzero spin is created by an elementary boson operator, $\psi_{m}$, with $Q=1$ and $F \neq 0$, with $m=-F, \ldots, F$.

General symmetry arguments can be used to deduce the low-energy theory coupling $\Psi$ to $\psi_{m}$ in the vicinity of the superfluid-insulator transition. For the $\Psi$ field alone, we have the familiar $|\Psi|^{4}$ field theory of the superfluidinsulator transition,

$$
\mathcal{S}_{\Psi}=\int d^{d} \mathbf{x} d \tau\left(|\partial \Psi|^{2}+r_{\Psi}|\Psi|^{2}+\frac{u_{\Psi}}{2}|\Psi|^{4}+\cdots\right)
$$

in $d$ spatial dimensions with co-ordinate $\mathbf{x}$, imaginary time $\tau$, and $(d+1)$-dimensional derivative $\partial^{2}=\partial_{\tau}^{2}+\nabla^{2}$. The even integer number of bosons per lattice site ensures that there is particle-hole symmetry in $\mathcal{S}_{\Psi}$ in the vicinity of the critical point.

The same particle-hole symmetry applies to the gapped $\psi_{m}$ field, but it is convenient to treat separately the particle-like (with $Q=1$ ) and hole-like (with $Q=-1$ ) 
excitations. The long-time behavior will be governed by those excitations just above the gap $\lambda$, for which the "nonrelativistic' limit can be taken and the action written

$$
\begin{array}{rl}
\mathcal{S}_{\psi}=\sum_{m} \int d^{d} \mathbf{x} & d \tau\left[p_{m}^{\dagger}\left(\mathrm{i} \partial_{t}-\frac{1}{2 m_{p}} \nabla^{2}+\lambda\right) p_{m}\right. \\
+ & \left.h_{m}^{\dagger}\left(\mathrm{i} \partial_{t}-\frac{1}{2 m_{h}} \nabla^{2}+\lambda\right) h_{m}\right],
\end{array}
$$

where $p_{m}$ and $h_{m}$ are fields describing particle and hole excitations of $\psi_{m} \sim u p_{m}+v(-1)^{F+m} h_{-m}^{\dagger}$, where $u$ and $v$ are coefficients chosen to that there is no $p_{m} h_{m}$ term in $\mathcal{S}_{\psi}$. The even integer density constraint requires that the gap $\lambda$ be the same in the particle and hole sectors, but the masses $m_{p, h}$ are, in general, allowed to be different.

The coupling between $\Psi$ and $\psi_{m}$ allows the conversion of a particle to a hole along with the creation of a pair:

$$
\mathcal{S}_{g}=g \sum_{m} \int d^{d} \mathbf{x} d \tau\left(\Psi^{\dagger} h_{m}^{\dagger} p_{m}+p_{m}^{\dagger} h_{m} \Psi\right)
$$

Our central results for the gapped excitations at the superfluid-insulator transition follow from the quantum field theory defined by $\mathcal{S}_{\Psi}+\mathcal{S}_{\psi}+\mathcal{S}_{g}$, and we will describe its properties below.

However, before we do so, we note that the same theory applies also to the superfluid-insulator transitions of fermions, with an even integer number of fermions per site: the only change is that the single particle excitations $p_{m}, h_{m}$ are fermionic. For a derivation for $F=1 / 2$ electrons, note that the filling $N=2$ per site requires the underlying model to have at least two bands to prevent a trivial filled-band ground state. Suitable orthogonal linear combinations of the electron annihilation operators in these bands yield the $p_{m}$ and $\varepsilon_{m m^{\prime}} h_{m^{\prime}}^{\dagger}$ respectively. $(\varepsilon$ is the $2 \times 2$ antisymmetric tensor).

We are interested here in the spectral functions of gapped excitations created by exciting a finite number of $p$ and $h$ quanta: at $T=0$, these will have thresholds in the spectral density at integer multiples of $\lambda$. As in previous work on spin ordering transitions [16], we now demonstrate that the structure of these threshold singularities reduces to the solution of an associated quantum impurity problem.

$\mathcal{S}_{\Psi}$ is isotropic in $d+1$ space- time dimensions, and so, at its critical point, is invariant under scaling transformations with dynamic exponent $z=1$. When such a rescaling transformation is applied to $\mathcal{S}_{\psi}$, it is clear that the coefficients of the dispersion $\left(2 m_{p, h}\right)^{-1}$ have scaling dimensions -1 , and are therefore irrelevant. The critical behavior is therefore given by $\left(2 m_{p, h}\right)^{-1}=0$, which describes a static impurity coupling to the bulk theory of $\mathcal{S}_{\Psi}$.

Applying the same scaling analysis to the coupling in $\mathcal{S}_{g}$ shows that the scaling dimension of $g$ is $(3-d) / 2$, so that it is relevant for $d<3$. Below we will address the case of two (spatial) dimensions, and describe an expansion in $\epsilon=3-d$ for the critical exponents.

We concentrate first on single-particle excitations with charge $Q=1$ and spin $F$. On the insulating side of the transition, where $r_{\Psi}>0$, the Green function for the $\psi_{m}$ field, $G^{\psi}$, will have a pole corresponding to the stable excitation at frequency $\omega=\lambda$, and a continuum for $\omega>\lambda+\sqrt{r_{\Psi}}$, at frequencies large enough for a particle to produce a hole along with a pair. As the transition is approached, $r_{\Psi}$ will become smaller while $\lambda$ remains fixed, and the continuum will start closer to the pole. On the superfluid side, the action described in $\mathcal{S}_{\Psi}$ must be rewritten in terms of amplitude and phase modes of the condensate. Away from the transition, the amplitude mode remains gapped, while the phase mode is the gapless Goldstone mode corresponding to broken phaserotation symmetry in the superfluid. Coupling between this gapless mode and $\psi_{m}$ will cause the continuum in $G^{\psi}$ to appear for frequencies just above $\lambda$. The quasiparticle pole remains, however, due to the factors of momentum appearing in the matrix element for coupling to the Goldstone mode, which cause the scattering rate to decrease as some power of $\omega-\lambda$.

Exactly at the transition, by contrast, the coupling is not restricted by the Goldstone theorem and the critical $\Psi$ excitations qualitatively modify the structure of $G^{\psi}$. The same is true of higher-order correlation functions, such as that for $p_{m} h_{m^{\prime}}$, which is the simplest spincarrying but charge-neutral combination.

These correlation functions can be found using a renormalization group (RG) calculation based on the formulation as a quantum impurity problem. Our approach is to perform a rescaling, which leaves $\mathcal{S}_{\Psi}$ invariant, to relate the correlation function evaluated at a frequency just above the gap, say $\omega=\lambda+\delta \omega$, to another frequency, $\omega^{\prime}=\lambda+\mathrm{e}^{-\ell} \delta \omega$, where $\ell$ is infinitesimal. The form of the coupling $\mathcal{S}_{g}$ allows the scale-invariance of $\mathcal{S}_{\Psi}$ to be used to relate correlators at these two frequencies.

As in the standard RG, the second stage of the calculation involves restoring the momentum cutoff to its original value. To lowest order in the coupling $g$ (or, as will subsequently be shown to be equivalent, in an expansion in $\epsilon=3-d$ ), the only diagram that must be calculated for the renormalization of $G^{\psi}$ is the self-energy diagram

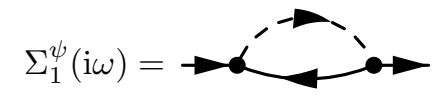

where the solid lines represent $\psi_{m}$ and the dashed line $\Psi$. The constant part of the diagram $\Sigma_{1}^{\psi}(\mathrm{i} \omega)$, along with a lower-order 'tadpole' diagram that we have omitted, produces a renormalization of the gap $\lambda$, but this is of no interest here.

It happens that there are no diagrams renormalizing the coupling $g$ at this order, although such diagrams do appear at higher order, and can be computed as in Ref. [17]. 
Since $\psi_{m}$ has only gapped excitations, it has no effect on the bulk scaling, which is therefore given by the standard results for a complex $|\Psi|^{4}$ theory. Rescaling by the standard phase space factor $\hat{u}=u_{\Psi} / S_{d+1}$ (where $S_{d}=2 /\left(\Gamma(d / 2)(4 \pi)^{d / 2}\right)$ ), there is a fixed point with coupling $\hat{u}=\epsilon / 5+\mathcal{O}\left(\epsilon^{2}\right)$ in $3-\epsilon$ dimensions, at which the scaling dimension of the field is given by $[\Psi]=1-\frac{\epsilon}{2}+\epsilon^{2} / 100+\mathcal{O}\left(\epsilon^{3}\right)$.

These results for the bulk, along with the self-energy diagram above, when extended to two loops, lead to the RG flow equation for the coupling to the 'impurity':

$$
\frac{\mathrm{d} \hat{g}}{\mathrm{~d} \ell}=\frac{\epsilon}{2} \hat{g}-\hat{g}^{3}+2 \hat{g}^{5}-\frac{\hat{u}^{2} \hat{g}}{4}-\frac{2 \pi^{2} \hat{u} \hat{g}^{3}}{3}+\mathcal{O}\left(\hat{g}\left(\hat{u}, \hat{g}^{2}\right)^{3}\right)
$$

where we have defined $\hat{g}=g(4 \pi)^{(d+1) / 2} / \Gamma((d-1) / 2)$. The coupling therefore approaches a fixed-point value with $\hat{g}^{2}=\epsilon / 2-\left(\pi^{2} / 15-49 / 100\right) \epsilon^{2}+\mathcal{O}\left(\epsilon^{3}\right)$, so that the perturbative expansion at this point is indeed equivalent to an expansion in $\epsilon$.

Finally, using the wavefunction renormalization of $\psi_{m}$, we arrive at the rescaling of the Green function $G^{\psi}$, which obeys

$$
G^{\psi}(\lambda+\delta \omega)=\mathrm{e}^{-y \ell} G^{\psi}\left(\lambda+\mathrm{e}^{-\ell} \delta \omega\right),
$$

where $y=1-\hat{g}^{2}+\hat{g}^{4}+\mathcal{O}\left(\hat{g}^{6}\right)$, with $\hat{g}$ set to the fixed point of Eq. (11); this yields $y=1-\epsilon / 2+\left(6 / 25-\pi^{2} / 15\right) \epsilon^{2}+$ $O\left(\epsilon^{3}\right)$. This can be iterated to give $G^{\psi}(\lambda+\delta \omega) \sim \delta \omega^{-y}$. Note that, at least to this order, $y<1$, so that the quasiparticle pole at $\omega=\lambda$ is replaced by a power-law threshold singularity. This exponent, determining the spectral density of the 'photoemission' of a single hole or particle at a band minimum or maximum, is one of our central results.

A similar calculation applies to the two-particle threshhold singularity at $\omega=2 \lambda$. This is associated with the renormalization of the $T_{m m^{\prime}} p_{m} h_{m^{\prime}}$ correlation function, where $T_{m m^{\prime}}$ is an arbitrary matrix. It is then also necessary to calculate the following insertion diagram (at one loop order):

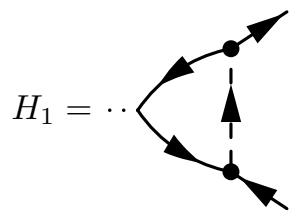

which accounts for the renormalization associated with bringing the particle and hole operators to the same space-time point. The resulting calculation is very similar and leads to the result for the two-particle propagator

$$
\Pi(\omega, \mathbf{k}=0) \sim(2 \lambda-\omega)^{-y_{2}}
$$

where the exponent $y_{2}$ depends upon whether the ma$\operatorname{trix} T_{m m^{\prime}}$ is symmetric or antisymmetric. For bosons, a symmetric $T$ has $y_{2}=1-2 \hat{g}^{2}+\mathcal{O}\left(\hat{g}^{6}\right)$, while an antisymmetric $T$ has $y_{2}=1$ exactly; these exponents therefore determine the threshold singularities for excitations with even and odd total spin $F$, respectively. For fermions, the same results apply, but with the roles of symmetric/antisymmetric $T$ reversed.

This paper has presented results for a variety of threshold singularities in the spectral functions of a system undergoing a superfluid-insulator transition, with an even number of particles per unit cell. This has direct application to experiments on ultracold atoms and to the cuprate compounds. It would be interesting to extend these methods to study non-equilibrium phenomena, such as those measured in Ref. 7], by methods explored in recent studies of non-equilibrium quantum criticality [18, 19] (which have so far been limited to $1+1$ dimensions).

We thank E. Demler and R. Shankar for useful discussions. This research was supported by the NSF grants DMR-0537077, DMR-0342157, and DMR-0354517.

[1] J. Stenger et al., Nature 396, 345 (1998).

[2] M. S. Chang et al, Phys. Rev. Lett. 92, 140403 (2004).

[3] J. M. Higbie et al, Phys. Rev. Lett. 95, 050401 (2005).

[4] M. S. Chang et al, Nature Phys. 1, 111 (2005).

[5] J. Mur-Petit et al, Phys. Rev. A 73, 013629 (2006).

[6] J. Kronjäger et al, cond-mat/ 0509083

[7] A. Widera et al, Phys. Rev. Lett. 95, 190405 (2005)

[8] F. Gerbier et al, Phys. Rev. A 73, 041602 (2006).

[9] H.-J. Huang and G.-M. Zhang, cond-mat/0601188

[10] A. Imambekov, M. Lukin, and E. Demler, Phys. Rev. A 68, 063602 (2003).

[11] S. Tsuchiya, S. Kurihara, and T. Kimura, Phys. Rev. A 70, 043628 (2004).

[12] D. Rossini et al, J. Phys. B: At. Mol. Opt. Phys. 39, S163 (2006).

[13] L. Zawitkowski et al, cond-mat/0603273

[14] R. Barnett, A. Turner, and E. Demler, cond-mat/ 0607253

[15] A. Rusydi et al, cond-mat/0604101

[16] S. Sachdev, M. Troyer, and M. Vojta, Phys. Rev. Lett. 86, 2617 (2001).

[17] S. Sachdev, Physica C 357, 78 (2001).

[18] K. Sengupta, S. Powell, and S. Sachdev, Phys. Rev. A 69, 053616 (2004).

[19] P. Calabrese and J. Cardy, Phys. Rev. Lett. 96, 136801 (2006). 